\title{
Rescuing Lives: When Cardiology Interweaves with Cognitive Neuroscience
}

\author{
Ahmad Yousef ${ }^{1}$ \\ 'School of Computational Science and Engineering, McMaster University, Hamilton, Ontario, Canada \\ *Correspondence: mohamas2@mcmaster.ca
}

Significant increase in the absolute power of gamma waves neural activities $(25-55 \mathrm{~Hz})$, after cardiac arrest in rats (11 seconds - 30 seconds) in the frontal lobes, the parietal lobes, and the occipital lobes. The previously mentioned finding hypothesizes that cardiac arrest causes greater consciousness than normal one, Borjigin, etal., 2013. In short, they suggested that there is high conscious neural activities after cardiac arrest, consciousness that we hypothesized to be flashback consciousness.

In this article, we will try to explain whether there are coherent communications between the heart and the brain (namely, the correlation between the conscious neural activities and the cardiac activities), whether the communications between the two organs are through physical neuronal connections, and/or virtual connections (quantum entanglement). We will also try to explain whether scientists can find ways to manipulate those communications to medically rescue critical cases though answering the following questions: how to avoid brain damages after cardiac arrest, how to overcome the cardiac arrest, and how to recover vegetative state.

We will start answering our first proposed question with the following hypothesis, deceleration of the heart rate causes greater conscious neural activities (flashback consciousness). Now, let us testify our speculation by reviewing the literature, and stating the most important points, as follows:

1- Vagus nerve stimulation temporarily causes cardiac arrest 'used for surgical purposes', Matheny etal., 1997.

2- Vegetative state patients have higher heart rates (90.0 bpm) than normal conscious people (78.5 bpm), Pattoneri etal., 2005.

3- Vagus nerve stimulation can be utilized to restore consciousness for vegetative state patients, Corazzol etal., 2017. In another word, heart rate deceleration assists in consciousness restoration, by activating the 'gamma waves' neural networks in the brain.

4- Sustained visual attention significantly decelerates heart rate, Richards et al., 1991. It, however, increases conscious 'gamma waves' neural activities.

5- Significant reduction in heart rate is occurred when human subjects view natural images, Laumann et al. 2003. We have to stop here for a moment; it seems to be we should expose the vegetative state patients to constantly view natural scenes to reduce their heart rate. Interestingly, natural scenes increase the dominance duration in binocular rivalry; Baker, etal. 2009. Natural scenes also recover Acute-Mental Stress; Brown, et al., 2013. Conclusively, natural images seem to increase the human automatic 'coherent' visual attention, that increases the activities of parasympathetic nerve system, and the conscious brain activities.

6- The power of gamma neural activities are greatest during theta troughs and lowest during theta peaks, von Stein, et al., 2000. Theta waves are known for actively motor behaviors, that activates the sympathetic nerve system, in turn, it will significantly increase the heart rates.

7- It has been documented that the plasma epinephrine concentration is high in isoflurane anesthesia resulting in higher cardiac sympathetic activity in human, Koitabashi et al, 1996. Picker et al. also showed that isoflurane decreased the vagal tone in dog, namely, increased heart rate. The aforementioned fact may explain why postcardiac arrest brain activities have the greatest absolute power for gamma waves, but the lowest for theta waves, see Borjigin, etal., 2013.

Collectively, the previous seven points might allow us to speculate and say the following: Cardiac activities are negatively correlated with conscious (gamma waves) neural activities. The maximal perceptual consciousness (flashback information) might be after the cardiac arrest (zero cardiac'activities), however, the minimal perceptual 
consciousness (information) might be synchronized with the maximal cardiac activities (vegetative state). We therefore hypothesize the following: inhibiting the 'gamma waves' neural networks; and stimulating the 'theta waves' neural networks, might be an integrative solution to recover cardiac arrest. Namely, in addition to defibrillators that might activate the heart after arrest; practitioners may require to use 'special kinds of transcranial brain stimulators' to disallow the neural spike trains from reaching the NCC neurons of following brain areas; the frontal lobes, the parietal lobes, the occipital lobes. Specifically, it is likely required to send resonance pluses to activate the inhibitory neurons of the feeders of the aforementioned brain regions. The purpose of these inhibitory processes is to reduce the patient's consciousness by deactivating its 'gamma waves' neural network, hopefully, the 'theta waves' network will be activated, and therefore the heart may beat again. Techniques of how to offer efficient stimulations for each case will be presented in a further research but see ref. 10 .

The conductivity between the heart and the brain requires more clarification. Cardiopulmonary nerve seems to be directly connected the 'theta waves' neural network in the brain. Vagus nerve, however, is tightly connected with the 'gamma waves' neural networks in the brain. It seems that most of the communications are achieved through physical connections (neurons); however, further research must be conducted to verify whether quantum entangled communications are existed as integrative contributors to interpret any instantaneous communications between the heart and the brain.

Ultimately, a balance between our detectable physics (physiology), and extra dimensional physics (consciousness) 'which is hypothesized to reside in extra physical dimensions (see reference 11)' should be stabilized to permit us stay as 'living entities'. Consistently speaking, we are in disagreement with scientists who think that the anatomical pathway of the recurrent laryngeal nerve (a branch of the vague nerve) is not optimal; we think however, its structure might refer to undiscovered communications between the nerve and the heart, communications that mostly utilize tachyons; and if this is the case, the receptive living biosensors of the aforementioned particles have to be in nanoscale, see the mathematical description of tachyonic dynamics, the superstring theory, and reference 22. Noticeably, the aforementioned nerve orders the vocal cord to abduct in inspiration, and adduct in expiration, namely, that nerve might have significant contributions in the respiratory sinus arrhythmia process (an accommodation process that accelerates heart rate for the inspiration and decreases it for the expiration). We are here taking about a network that might "tightly" connect the heart with the brain; fulsome understanding of this network may enable us to deeply decipher the secrets of our recent findings on how the deep breathing can alter visual motion perception and bistable auditory perception, see reference 12 and 13. Interestingly, play-backward perception are produced through the expiration, as if the deceleration of heart rate produces 'play-backward' consciousness.

Important to mention, several studies have reported that the systole versus diastole in the cardiac cycle impacts the perception of visual or auditory stimuli, see reference 14 and 15, (but also see references 16 and 17 for nonsignificant heart phase dependent effects). The clear distinction of the perceptual alteration, however, was recently reported to be the breathing cycle not the cardiac cycle; we therefore advice the scientists to zoom out and have a global view to see what essentially makes the heart alter the perception.

The answer exclusively seems to be "the massive changes in the heart rate"; and this can be noticed through our recent reports, see reference 12 and 13; namely, because the fellows do not consider the respiratory sinus arrhythmia process; their report show noticeable amount of false alarm and missed detection of the tactile stimulation as reported by their human subjects, see reference 18. Although their 'zoom in' investigation is really fulsome, but solid research shall also have 'zoom out' investigations; namely, to consider respiratory sinus arrhythmia process in their further studies. In another word, we have to emphasize that, unlike the perceptual rivalries which are reported through 'deep breathing research'; there are also 'mini-perceptual rivalries', and these rivalries are the main reason behind the inaccuracies in the detection rates; therefore, zoom-out investigations become a must!

There is a scientific common agreement that the average individual may detect the stimulus when it interacts with central sensory receptors way easier than peripheral ones (see our theory of two integrative conscious brains in reference 21). Interestingly, Esra, etal. found that the low, but not the high, heartbeat evoked potentials (HEPs) generate better detection rates; namely, HEPs are inversely correlated with the detection rates. The previous finding is astonishing; presumably because the heart rate is inversely correlated with the HEPs in healthy individuals as well as sleep disordered breathing patients, see references 19 and 
20; thus, the detection rate shows direct correlation with the heart rate. Considering the theory of two integrative conscious brains, 'the central conscious brain' is active when the heartbeat evoked potentials have low amplitudes (high heart rate), and thus, high detection rates in favor of the external environment but reduced level of the inner $\&$ flashback consciousness; more reduction in the HEPs amplitudes may enter the subject into unrecovered vegetative state, see reference 12 (page 3 ) for more information.

We therefore have to emphasize that scientists shall search for a stable avenue that neither resides in the vegetative state as a result of higher heart rates, nor potentially invoked-playback of the inner conscious movie as a result of the cardiac arrest.

Has to be emphasized, our hypothesis about the inverse correlation between the total brain gamma neural activities (including but not limited to activities from the frontal lobes, the parietal lobes, the occipital lobes) versus the cardiac activities is really clear in several undoubtable circumstances; for instance, increasing the conscious cortical activities, by stimulating the Vagus nerve, shall signal both the SA and AV nodes by releasing the neurotransmitter acetylcholine that slows the heart rate by opening ligandgated potassium ion channels to slow the rate of spontaneous depolarization, which extends repolarization and elongates the time before the next automatic depolarization occurs at the neuromuscular junction. Due to the sophistication of that network, further discussion will be offered in a following research.

To summarize the purpose of this article, we cautiously advise the scientists and the health care professionals to consider the application of Simultaneous heart-brain network manipulations for the patients in critical cardiac or consciousness states, Precise simultaneous manipulations, stimulation/inhibition, to various areas in the brain and in the heart, that comply to the seven aforementioned points; may allow the practitioners not only to rapidly restore consciousness to vegetative state patients, but to break the cardiac arrest without brain damage. Ultimately, this technique may not only elongate people live, but intensify their 'living' but not 'flashback' consciousness.

Essential Notification

Scholars with brain imaging, ECG, and professional eyetracker facilities are welcome to collaborate in challenging investigations, namely, to investigative every possible communication between the heart and the brain in a fulsome way. Enquires should be directly sent to the author.
Transactional References

[1] Borjigin, etal., (2013). "Surge of neurophysiological coherence and connectivity in the dying brain" PNAS, 2013.

[2] Matheny etal., (1997). "Vagus nerve stimulation as a method to temporarily slow or arrest the heart". Annals of thoracic surgery.

[-] Corazzol etal., (2017)"Restoring consciousness with Vagus nerve stimulation". Current Biology.

[3] Pattoneri etal., 2005. "Circadian blood pressure and heart rate changes in patients in a persistent vegetative state after traumatic brain injury". The Journal of Clinical Hypertension.

[4] Richards et al., (1991). "Heart rate variability during attention phases in young infants". Psychophysiology.

[5] Brown, et al. (2013). "Viewing Nature Scenes Positively Affects Recovery of Autonomic Function Following Acute-Mental Stress". Environmental Science and Technology.

[6] Laumann et al. (2003). "Selective attention and heart rate responses to natural and urban environments". Journal of Environmental Psychology.

[7] von Stein, et al., (2000). "Top-down processing mediated by interareal synchronization". PNAS Biological Sciences.

[8] Koitabashi etal. "The effect of volatile anesthetics on plasma-free catecholamine concentrations during surgery. JJSCA 1996.

[9] Picker etal. "Inhalation anaesthetics increase heart rate by decreasing cardiac vagal activity in dogs". Br J Anaesth 2001. [10] Y. Wang and L. Guo, "Nanomaterial-enabled neural stimulation," Frontiers in Neuroscience, vol. 10, 2016.

[11] Yousef, A. "Consciousness Might Be Localized in Extra Physical Dimensions." PsyArXiv, doi:10.31234/osf.io/angc8.

[12] Yousef, A. 2019. "Deep Breathing Alters Visual Motion Perception.” PsyArXiv. doi:10.31234/osf.io/up3sa.

[13] Yousef, A. 2020. "Deep Breathing Alters Bistable Auditory Perception." PsyArXiv. doi:10.31234/osf.io/fv5ar.

[14] Saxon, Detection of near threshold signals during four phases of cardiac cycle. Ala. J. Med. Sci. 7, 427-430 (1970).

[15] Sandman etal. Heart rate and cardiac phase influences on visual perception. J. Comp. Physiol. Psychol. 91, 189-202 (1977).

[16] Elliott, and Graf, Visual sensitivity as a function of phase of cardiac cycle. Psychophysiology 9, 357-361 (1972).

[17] Delfini, and Campos, Signal detection and the "cardiac arousal cycle". Psychophysiology 9, 484-491 (1972).

[18] Esra Al, etal, 2020. Heart-brain interactions shape somatosensory perception and evoked potentials. Proceedings of the National Academy of Sciences.

[19] Konecny etal. 2021. Increased heart rate with sleep disordered breathing in hypertrophic cardiomyopathy. Int J Cardiol.

[20] Immanuel S., etal. 2014. Heartbeat evoked potentials during sleep and daytime behavior in children with sleep-disordered breathing. Am J Respir Crit Care Med.

[21] But, why two integrative conscious brains? https://www.youtube.com/watch?v=d9mAtxF5Z9Q

[22] Yousef, A. 2019. "Tachyons but Not Photons Might Generate Conscious Dreams.” PsyArXiv. doi:10.31234/osf.io/hvqy7. 